\title{
Cilia are required for asymmetric nodal induction in the sea urchin embryo
}

\author{
Matthias Tisler ${ }^{1}$, Franziska Wetzel $^{1 \dagger}$, Sabrina Mantino ${ }^{1 \dagger}$, Stanislav Kremnyov ${ }^{2}$, Thomas Thumberger ${ }^{1,3}$, \\ Axel Schweickert ${ }^{1}$, Martin Blum ${ }^{1}$ and Philipp Vick ${ }^{1 *}$ (1)
}

\begin{abstract}
Background: Left-right (LR) organ asymmetries are a common feature of metazoan animals. In many cases, laterality is established by a conserved asymmetric Nodal signaling cascade during embryogenesis. In most vertebrates, asymmetric nodal induction results from a cilia-driven leftward fluid flow at the left-right organizer $(\mathrm{LRO})$, a ciliated epithelium present during gastrula/neurula stages. Conservation of $L R O$ and flow beyond the vertebrates has not been reported yet.
\end{abstract}

Results: Here we study sea urchin embryos, which use nodal to establish larval LR asymmetry as well. Cilia were found in the archenteron of embryos undergoing gastrulation. Expression of foxj 1 and dnahy suggested that archenteron cilia were motile. Cilia were polarized to the posterior pole of cells, a prerequisite of directed flow. High-speed videography revealed rotating cilia in the archenteron slightly before asymmetric nodal induction. Removal of cilia through brief high salt treatments resulted in aberrant patterns of nodal expression. Our data demonstrate that cilia - like in vertebrates - are required for asymmetric nodal induction in sea urchin embryos.

Conclusions: Based on these results we argue that the anterior archenteron represents a bona fide LRO and propose that cilia-based symmetry breakage is a synapomorphy of the deuterostomes.

Keywords: Sea urchin, Deuterostomes, Left-right asymmetry, Symmetry breakage, Nodal, Cilia, Left-right organizer

\section{Background}

Vertebrates possess pronounced visceral asymmetries along their left-right (LR) body axis, although they belong to the large phylogenetic group of the Bilateria, which refers to their bilaterally symmetric outer appearance [1]. Most organs are positioned in a characteristic way in the thoracic and abdominal cavities. In all vertebrate species examined so far, these asymmetries are under the control of the Nodal signaling cascade, which is only activated in the left lateral plate mesoderm before the first appearance of anatomical asymmetries [2]. The secreted transforming growth factor beta (TGF $\beta$ ) Nodal binds to its receptor which results in the activation of its own transcription and that of its negative feedback inhibitor lefty (left-right determination factor), another

\footnotetext{
*Correspondence: philipp.vick@uni-hohenheim.de

${ }^{\dagger}$ Equal contributors

${ }^{1}$ University of Hohenheim, Institute of Zoology, 70593 Stuttgart, Germany

Full list of author information is available at the end of the article
}

secreted TGF $\beta$ superfamily member. Lefty antagonizes Nodal signaling, providing its temporal and spatial control. Additionally, the paired-like homeodomain transcription factor pitx 2 is induced downstream of Nodal and mediates, through less well-known target gene activation, the setup of asymmetric organ morphogenesis.

The event activating this highly conserved developmental program is referred to as symmetry breakage. Even though variations of the common theme may exist, an ancestral mode of vertebrate symmetry breaking has emerged over the past few years: at the heart of this mechanism acts an extracellular leftward fluid flow, generated by a transient ciliated epithelium, the so-called left-right organizer (LRO) [3-5]. The vertebrate LRO (known as Kupffer's vesicle in fish, gastrocoel roof plate (GRP) in amphibians, and posterior notochord or ventral node in mammals) represents a field of mono-ciliated cells at the posterior end of the forming notochord, flanked by endodermal archenteron cells [6-8]. This unique tissue, which develops during early neurula stages, consists of superficially located mesendodermal

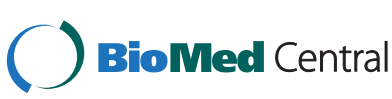

(C) 2016 The Author(s). Open Access This article is distributed under the terms of the Creative Commons Attribution 4.0 International License (http://creativecommons.org/licenses/by/4.0/), which permits unrestricted use, distribution, and reproduction in any medium, provided you give appropriate credit to the original author(s) and the source, provide a link to the Creative Commons license, and indicate if changes were made. The Creative Commons Public Domain Dedication waiver (http://creativecommons.org/publicdomain/zero/1.0/) applies to the data made available in this article, unless otherwise stated. 
cells which are transiently placed in the primitive gut or archenteron, where they function as the LRO. The cells of the LRO subsequently leave the epithelium to ingress into deeper mesodermal structures $[7,8]$. The polarized attachment of cilia at the posterior cell surface, together with their clockwise rotational movement, create an asymmetric stimulus by the leftward acceleration of extracellular fluids, i.e. a leftward flow [9]. This setup has been functionally described in mammals (mouse, rabbit), amphibians (Xenopus) and teleost fish (medaka, zebrafish), and homologous tissues have been identified in salamanders (axolotl) and basal bony fish (white sturgeon). Interference with ciliary length, motility, polarization or flow function in general resulted in defects of the LR axis [10-15].

As a result of leftward flow, the Nodal inhibitor dand5 becomes down-regulated on the left margin of the LRO, where it is co-expressed on both sides with nodal itself. Nodal thus becomes liberated on the left side to induce the asymmetric signaling cascade in the left lateral plate mesoderm [16-18]. dand5 repression is induced through a flow-dependent intracellular calcium signal, which is mediated through the calcium channel pkd2 (polycystic kidney disease 2). Although ubiquitously expressed during these stages, pkd2 inhibition causes LR defects and prevents a unilateral calcium signal in mouse and fish LROs [5, 19-22].

Outside of the vertebrate lineage, LR asymmetries are common as well. For many deuterostome lineages, unilateral nodal expression has been described, which seems to be the common mediator of LR asymmetries in metazoan animals [3, 23-25]. Tunicates, the sister group of the vertebrates, as well as the cephalochordates, the most basal group of chordates, both express nodal asymmetrically on the left side [26, 27]. The latter seem to show an ancient state of this set-up, with many LRO targets activated similarly to vertebrates, such as nodal, lefty, pitx 2 and dand5 [26, 28-31]. Accordingly, interfering with Nodal activity in the cephalochordate amphioxus also resulted in LR defects [32]. The existence of an LRO in amphioxus has been predicted, but not yet analyzed or functionally tested $[3,11]$. Within the deuterostomes, the Ambulacraria, a chordate sister group which comprises the echinoderms, show unilateral nodal activation during late gastrula stages as well [33]. Although adult echinoderms took the path of reestablishing a radially pentameric body plan during evolution, their early embryo displays the typical bilaterally symmetrical embryonic development, which results in a pronounced LR asymmetry before metamorphosis [34]. As in other deuterostomes, nodal is the first asymmetrically activated gene, and is first found on one side in the developing archenteron tip [33, 35]. The functional requirement and a complex downstream gene regulatory network have been elucidated in detail in sea urchin embryos [36]. This asymmetric archenteron domain instructs a second symmetrical ectodermal nodal domain to switch towards this side as well [37]. This expression is thought to be on the right, as deduced from a ventral mouth opening [25]. We have previously argued that this difference can be resolved to a common evolutionary origin, if the ventral side of echinoderms is homologized with the dorsal side of chordates [11]. This reasoning is based on the expression of dorsal organizer genes in the oral ectoderm of the sea urchin larva, suggesting that the mouth - counterintuitively - opens on the dorsal side. Such an evolutionary mouth repositioning can be easily envisaged as a transitional process from echinoderm ancestors to vertebrates [38]. Indeed, oral organizer identity has been recently shown to be functionally conserved in the sea urchin P. lividus [39]. Thus, in this scenario, left-asymmetric nodal expression is a synapomorphy of the deuterostomes [11].

One major question, however, has remained unanswered: how does symmetry breakage upstream of asymmetric nodal induction occur in echinoderms? Do sea urchin embryos possess a LRO or an evolutionary functional precursor that induces asymmetric nodal expression? Do archenteron cells possess cilia and, if so, are these required to induce asymmetric nodal expression? Using descriptive and functional approaches we show that (1) archenteron cells in the sea urchin larva harbor monocilia; (2) archenteron cilia are polarized and motile; and (3) cilia are required for asymmetric nodal induction.

\section{Results}

Sea urchin archenteron cells harbor polarized monocilia The defining feature of vertebrate LROs are polarized monocilia. As an entry point into studying sea urchin symmetry breakage, we investigated the presence of cilia on staged gastrula embryos by performing immunofluorescence (IF) with a well-characterized anti-acetylated $\alpha$-tubulin antibody. In addition to the previously described long ectodermal cilia, optical sections of gastrula stage Paracentrotus lividus embryos revealed a population of shorter cilia within the developing archenteron (Fig. 1a-b"). Higher magnification showed that most cilia ranged from $4-6 \mu \mathrm{m}$ in length, with the most anterior ones being longer, measuring up to $10 \mu \mathrm{m}$ (Fig. 1c). The same basic characteristics of mesendodermal archenteron cilia were also found in a second sea urchin species, Strongylocentrotus pallidus, demonstrating the conserved presence of cilia in the primitive gut of sea urchin larvae. One slight variation was observed, namely that very early gastrula embryos of Strongylocentrotus apparently lacked archenteron cilia (Fig. 1d), which were, however, present at mid to 


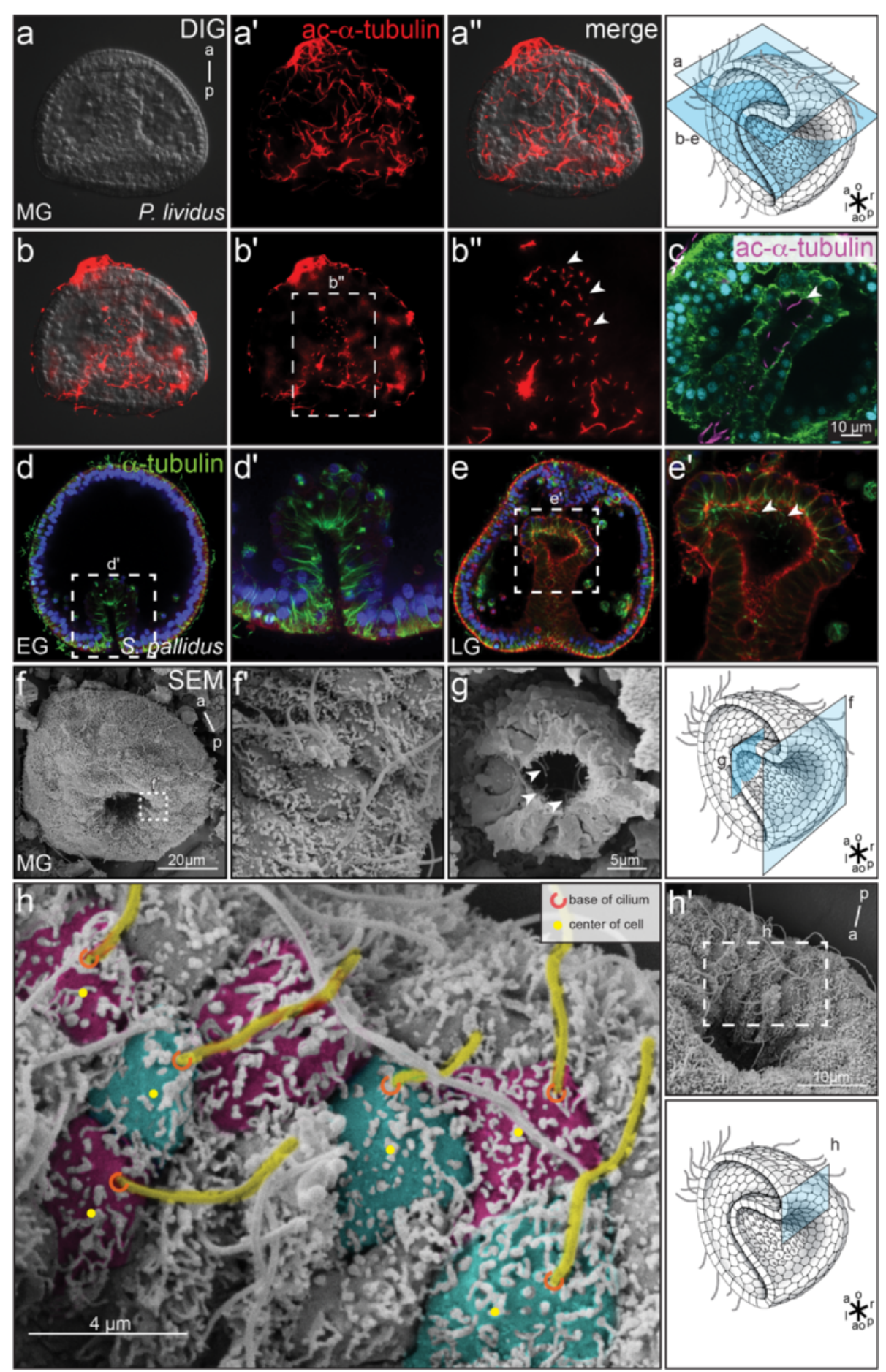

Fig. 1 Polarized cilia at the sea urchin archenteron. a-e $P$. lividus $(\mathbf{a}-\mathbf{c})$ and S. pallidus embryos (d-e') were analyzed by IF for the presence of cilia

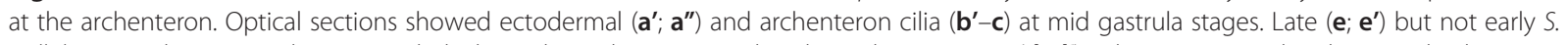
pallidus gastrula stage embryos revealed cilia in the archenteron and at the archenteron tip (d, $\mathbf{d}^{\prime}$ ). Cilia were stained with an antibody against acetylated-a-tubulin (red, $\mathbf{a}^{\prime}-\mathbf{c}$ ) or anti-a-tubulin (green, $\mathbf{d}-\mathbf{e}^{\prime}$ ), nuclei were stained with DAPI ( $\left.\mathbf{d}-\mathbf{e}^{\prime}\right)$, and cell boundaries were visualized by phalloidin-green (c) or phalloidin-red (d-e'). $\mathbf{f}, \mathbf{g}$ SEM analysis of $P$. lividus ectodermal and archenteron cilia. Fractured embryos allowed the visualization of monocilia on archenteron cells $(\mathbf{g})$. Cilia are highlighted by an arrowhead $\left(\mathbf{f}-\mathbf{h}^{\prime}\right)$ Posterior polarization of cilia on cells which invaginated into the archenteron. Cilia are colored in yellow and individual cells alternating in green and purple. The ciliary base is marked by a red semicircle, the center of the cell is indicated by a yellow dot. Schematic drawings adapted from Blum et al. 2014 [3] 
late gastrula stages (Fig. 1e). In P. lividus, cilia were present already at earlier gastrula stages (Fig. 1b, and data not shown).

Scanning electron microscopy (SEM) of P. lividus was employed to further characterize archenteron cilia and to test a potential polarization of cilia along the animalvegetal, and thus along the AP axis of the embryo (Fig. 1f-h). Embryos, which were broken perpendicularly to the animal-vegetal axis and thus allowed a highpower magnification view inside the archenteron, revealed monocilia of about $4 \mu \mathrm{m}$ in length (Fig.1f, g). Archenteron cilia were clearly present already in mid gastrula stage embryos (Fig. 1f). Importantly, using the cilium-insertion point and the cell center as reference points, a clear posterior polarization of cilia was obvious already when cells were orienting towards the inside of the archenteron in animal/anterior direction (Fig. 1h). In summary, our descriptive analysis of the archenteron showed that cells harbored monocilia at a time point just prior to the asymmetric induction of nodal, suggesting a functional role homologous to that of vertebrate LROs.

\section{The sea urchin gastrula embryo expresses marker genes} for motile cilia

Next we analyzed the expression of marker genes indicative of motile cilia. To that end, sea urchin homologs of two marker genes for motile cilia, dynein axonemal heavy chain 9 (dnah9) and forkhead box protein J1 (foxj1) were cloned by RT-PCR and expression patterns during embryonic development were assessed by wholemount in situ hybridization. In the ectoderm, dnah9 was broadly expressed with intense signals in the apical tuft region. Localized mRNA expression was also found in the vegetal part of the gastrula mesendodermal tissue, followed by expression in the archenteron (Fig. 2a, b). Sense control probes were negative at all stages examined and for all genes analyzed in this study (Fig. 2c and data not shown). Analyses of foxj1 mRNA expression revealed a similar pattern in the ectoderm and strong staining in the area of the developing apical tuft (Fig. 2e-g). At early gastrula stages, localized expression was found in the vegetal plate region (Fig. 2e), while in late gastrula stages, a mesodermal expression domain started to appear at the anterior tip of the archenteron (Fig. 2g). These expression patterns were indicative of a population of motile mesendodermal cilia in the archenteron and reminiscent of vertebrate LROs.

To investigate additional potentially conserved LRO genes, we cloned P. lividus Bicaudal C homolog 1 (bicc1) [40], which is required for cilia polarization at the vertebrate LRO, and $p k d 2$, a calcium channel required for sensing of the flow [22]. Expression of bicc1 has been recently described in the sea urchin Hemicentrotus

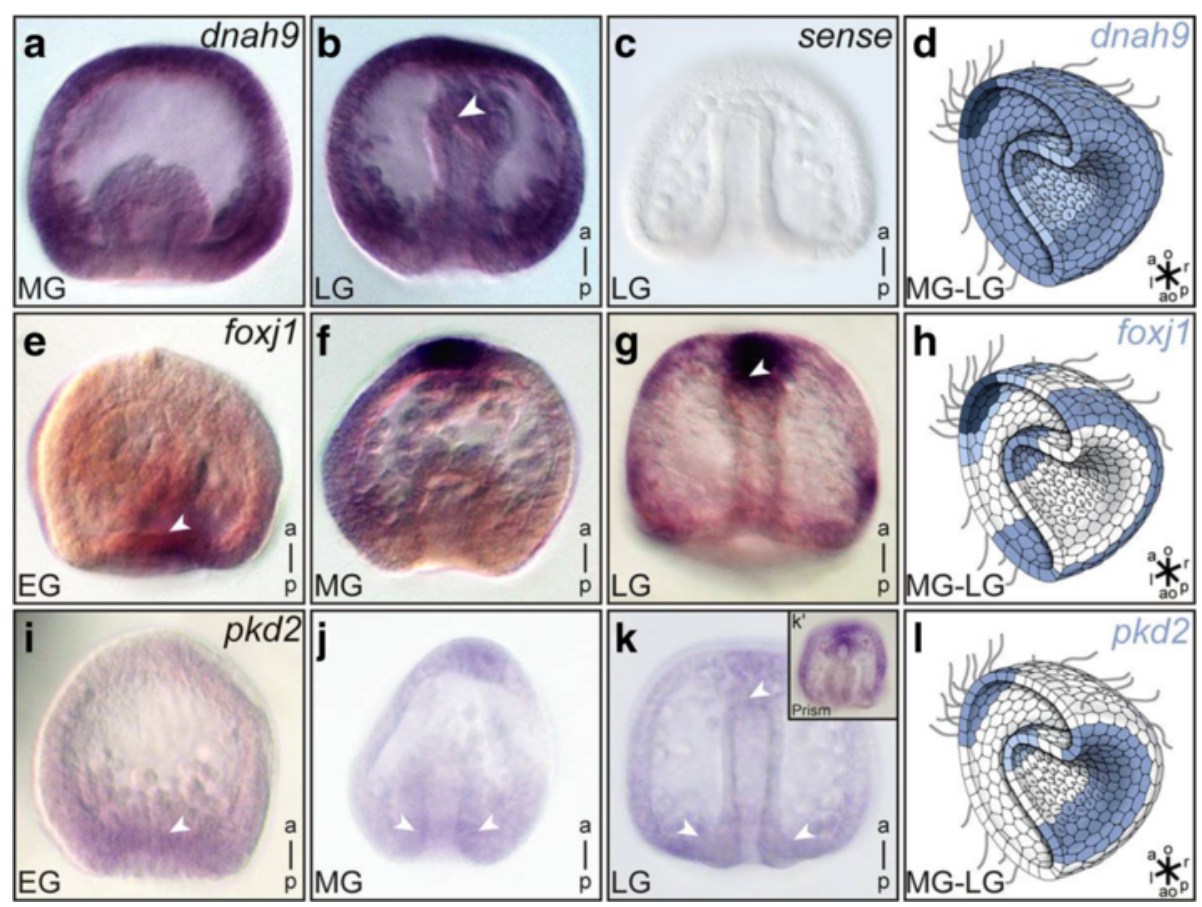

Fig. 2 Motile cilia marker genes dnah9 and foxj1 are expressed throughout sea urchin gastrulation. Whole mount in situ hybridization of early (EG), mid (MG) and late (LG) gastrula stage P. lividus embryos, as well as prism stages ( $\left.\mathbf{k}^{\prime}\right)$ for mRNA expression of dnah9 (a-d), foxj1 (e-h) and pkd2 (i-I). Schematic representation of staining in mid-gastrula embryos is highlighted in drawings in $(\mathbf{d}, \mathbf{h}$ and $\mathbf{I})$. White arrowheads highlight vegetal blastopore and archenteron tip expression areas. Schematic drawings adapted from Blum et al. 2014 [3] 
pulcherrimus, i.e. an unrelated genus. Our analysis in $P$. lividus confirmed the reported localization, i.e. strong expression of bicc1 mRNA at the vegetal pole of early to late gastrula stage embryos, directly at the site of invagination (Additional file 1: Figure S1 and [41]). P. lividus pkd2 mRNA (also termed suPC2) was expressed in a pattern reminiscent of bicc1 in early to late gastrula stages, namely in vegetal cells of the early gastrula embryo (Fig. 2i-l). pkd2 mRNA transcription was further activated in the apical tuft cells and at the archenteron tip of late gastrula to early prism stage embryos (Fig. 2i-k).

\section{Cilia in the archenteron are motile}

In order to directly assess the potential motility of archenteron cilia in live embryos, we analyzed early to midgastrula stage embryos by high-speed videography. To highlight moving objects, movies were processed using a temporal difference imaging method (cf. Material and Methods). Additional file 2: Movie 1 demonstrates that the posteriorly polarized cilia of invaginating archenteron cells (cf. Fig. 1g) were indeed highly motile. Next we analyzed mid to late gastrula stage embryos, focusing on the lumen of the central part of the elongated archenteron. Again, the monocilia detected by SEM analysis (Fig. 1h) were motile, displaying a rotating pattern (Additional file 3: Movie 2). Attempts to visualize a possible effect of cilia motility, i.e. whether or not this resulted in directed movement of extracellular fluids, failed due to technical reasons, as we were not able to introduce fluorescent micro beads into the archenteron (not shown). Despite this shortcoming, our results strongly suggest that the sea urchin embryo harbors a vertebrate-type LRO, i.e. an archenteron epithelium with posteriorly polarized rotating monocilia, which expresses a set of characteristic marker genes, including foxj1, dnah9, bicc1 and pkd2.

\section{Archenteron cilia are required for asymmetric nodal induction}

As an alternative to visualizing cilia-driven fluid flow, we chose to directly test the function of cilia in asymmetric nodal induction. In a first set of experiments we used the pharmacological inhibitor Ciliobrevin D, which inhibits the ATPase activity of axonemal and cytoplasmic dynein motor proteins [42]. Treatment of early gastrula stage embryos with $50 \mu \mathrm{M}$ Ciliobrevin D efficiently inhibited ciliary motility, as judged by direct microscopic observation of embryonic swimming behavior, which completely ceased within minutes (data not shown). As this treatment efficiently prevented gastrulation movements as well, we were not able to analyze later nodal expression (data not shown).

Short of a specific inhibitor of axonemal dynein function, we decided to assess the role of cilia for symmetry breakage by removing cilia from the embryo. To that end, a brief osmotic shock with high-salt (HS) seawater containing twice the normal molarity of $\mathrm{NaCl}$ (sodium chloride) was applied, a procedure previously reported to completely deciliate the ectodermal surface of the embryo [43]. Embryos between early gastrula and prism stage were treated with HS seawater for $60-90 \mathrm{~s}$ and returned to regular seawater until untreated control embryos reached late gastrula to pluteus stages. Embryos were fixed, assessed for developmental defects and/or processed for IF, SEM or in situ hybridization. First we analyzed whether this procedure was suited to remove archenteron cilia. Swimming behavior was instantly impaired, as described for Ciliobrevin above (not shown). Embryos were fixed $20 \mathrm{~min}$ after treatment and subjected to IF analysis of cilia or SEM analysis. While normal ciliation was seen in SEM photographs of untreated embryos, HS-treated specimens were devoid of cilia, both on the outer surface and at the proximal (posterior) end of the archenteron cavity (Fig. 3a, b). To investigate whether cilia were removed along the entire archenteron, we analyzed optical sections of treated embryos using IF. Control embryos displayed normal ciliation along the extended archenteron. In contrast, about half of the HS-treated embryos lacked cilia altogether, with the remainder of specimens displaying a small number of very short cilia remnants (Fig. 3c, d; Additional file 1: Figure S1i). These experiments demonstrated that HS treatment was an efficient tool to remove archenteron cilia from the embryo.

Because of the proven function of cilia in signal transduction in different species $[44,45]$, we asked whether deciliation affected normal embryonic development. The protocol applied here has been previously used to remove ectodermal cilia at different stages of development, without any reports of developmental defects [46-49]. Stephens et al. (1977) even applied multiple rounds of deciliation (up to ten times) which resulted in phenotypically normal pluteus larvae [50]. In order to confirm that the HS-protocol did not interfere with normal embryogenesis in our experiments as well, $>1.500$ embryos were deciliated in 11 independent experiments at different time points during development, between late blastula and late gastrula stages. Treated specimens were fixed and assessed for developmental delays or phenotypic alterations at time points when untreated control embryos had reached a) late gastrula with fully elongated archenteron; or b) early pluteus stages. In both sets of experiments, no phenotypical difference was noted between control and HS-treated samples (Additional file 1: Figure S1e-h). Quantitative evaluation of experiments revealed no temporal delay in either of these groups, besides minor and random fluctuations that were sometimes observed (Additional file 1: Figure S1j). Together 


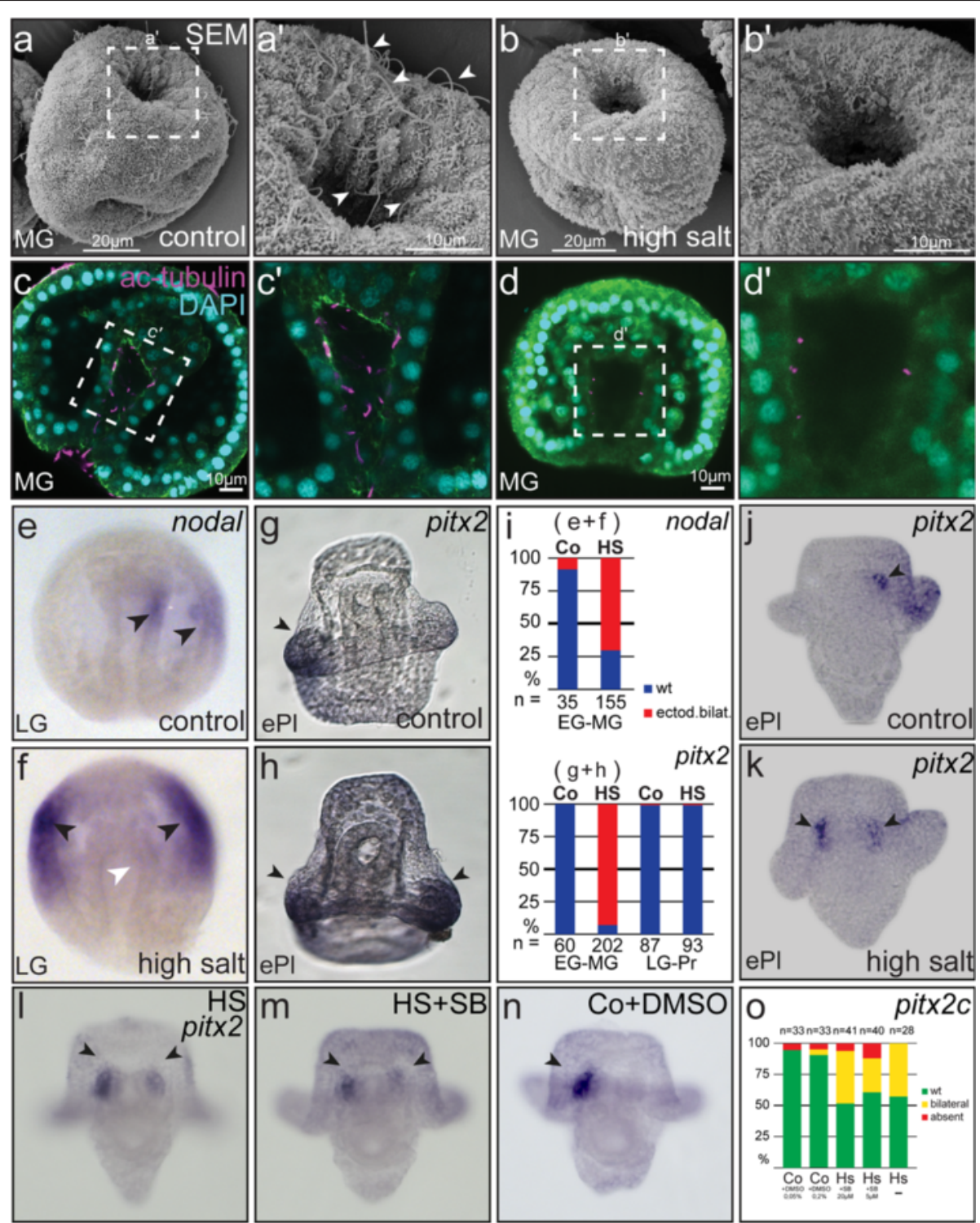

Fig. 3 Deciliation impairs LR asymmetric nodal cascade induction. SEM (a, b) and IF (c, d) analyses of cilia in control untreated embryos (a, $\mathbf{c})$ and specimens exposed to a 60-90 s osmotic shock (b, d). Note that cilia were almost completely absent following high salt treatment. Cilia in (c) and (d) were stained with an antibody against anti-acetylated-a-tubulin (purple), nuclei with DAPI, and cell boundaries visualized by phalloidin-green. $\mathbf{e}, \mathbf{g}$ Unilateral induction of asymmetric nodal cascade genes in control embryos. $\mathbf{f}, \mathbf{h}$ Bilateral ectodermal nodal and pitx2 expression following deciliation of early to mid-gastrula stage embryos. Black arrowheads highlight expression in the archenteron and ectoderm; white arrowheads indicate lack of expression. i Quantification of expression pattern from (e-h). Unilateral pitx2 expression in coelomic pouches of pluteus stage control specimen (j) and bilateral expression in deciliated embryos (k). Bilateral expression of pitx2 after deciliation is independent of MAPK/p38 inhibition through SB203580 (I-m). o Quantification of expression patterns. Posterior/vegetal is to the top in $(\mathbf{a}, \mathbf{b})$ and to the bottom in $(\mathbf{c}-\mathbf{g})$

with the previously published deciliation approaches, these control experiments demonstrate that the protocol applied here efficiently removed archenteron cilia without impairment of normal development.

In a final series of experiments, we tested whether deciliation impacted on nodal asymmetry in the archenteron and ectoderm, which we hypothesized based on our descriptive analysis of archenteron cilia. HS treated embryos indeed revealed grossly altered patterns of nodal expression at late gastrula stages, when control embryos displayed unilateral patterns in both, the archenteron and the ectoderm (Fig. 3e, f). Surprisingly, HS treatment at early to mid-gastrula stages, i.e. just before asymmetric induction of the nodal cascade, resulted in an expanded, bilateral ectodermal expression of nodal in the vast majority of treated specimens. The same result was obtained with pitx2 (Fig. 3g, h), a direct target of Nodal [51]. Bilateral activation of the Nodal cascade was 
especially evident when analyzed in the developing coelomic pouches of early pluteus stage embryos, using pitx 2 as a late LR marker gene (Fig. 3j, k). The archenteron domain of nodal in late gastrula stage embryos, however, was mostly undetectable (Fig. 3e, f).

Next we tested whether high-salt treatments resulted in a stress-induced activation of MAPK/p38-mediated ectopic activation of nodal [52]. If it were, inhibition of MAPK/p38 should rescue the deciliation-induced aberrant activation of the Nodal cascade. Blastula-stage embryos were treated with the MAPK/p38 pathwayspecific inhibitor SB203580 [52], to test the efficiency of the drug, which resulted in dorso-ventral axis defects (Additional file 1: Figure S1k-n). When embryos were treated after deciliation at mid to late gastrula stages, development was not impaired but specimens still exhibited bilateral pitx2 expression in the coelomic pouches at early pluteus stage (Fig. 3l-o). These experiments thus demonstrated that the expanded expression pattern of LR marker genes after deciliation was not due to MAPK/p38-mediated over-activation of Nodal signaling during gastrulation.

In order to determine whether there was a sensitive time period, i.e. whether cilia were required only during certain developmental stages, we performed a time course of deciliation and treated specimens with high salt at defined points of development: early gastrula, mid gastrula, late gastrula or prism stage. Based on ciliadriven symmetry breakage in the vertebrates, the time window was expected to be rather narrow. Figure $3 \mathrm{i}$ demonstrates that deciliation at very early to midgastrula stages, before full extension of the archenteron and before asymmetric nodal expression, caused aberrant LR development. Embryos treated after this point, during late gastrula to early prism stages, showed normal unilateral expression of nodal, i.e. the sensitive time window closes during late gastrulation.

In summary, our work demonstrates that the sea urchin archenteron harbors polarized and motile monocilia, which are required for LR axis determination during gastrulation.

\section{Discussion}

Theoretical considerations and deductive logics have previously led us to propose that sea urchin embryos possess an ancestral LRO, homologous to that of the vertebrates [11]. Here we demonstrate in two species that sea urchin gastrula embryos indeed display a mesendodermal monociliated archenteron, which is reminiscent of the vertebrate LRO (Fig. 4a). Importantly, cilia were required for asymmetric nodal induction, arguing for a conserved cilia-based mechanism for LR symmetry breakage in the deuterostome lineage.
Common features between the sea urchin archenteron and vertebrate LROs include polarized and motile monocilia as well as expression of dnah 9 and foxj1, two genes which are mandatory for generation of leftward flow. Although we were not able to visualize a fluid flow within the archenteron directly, we propose that ciliadependent symmetry breakage is a conserved feature between sea urchin and the vertebrates. In order to qualify as the nodal inducing event, cilia-driven symmetry breakage should occur at the right time, before asymmetric nodal induction, and in the right place, i.e. at the archenteron. Besides the presence of motile cilia itself, the expression of vertebrate LRO components dnah9, foxj1, and $p k d 2$ in this very region are in perfect agreement with this proposal (Fig. 2).

Not every LRO feature seems to be conserved between sea urchins and the vertebrates, though. While flowperceiving (and nodal expressing) cells in vertebrates are located at the posterior pole of the embryo, close to the blastopore or proximal end of the gut, these cells reside at the anterior tip of the archenteron in the sea urchin late gastrula embryo, at its distal end, as deduced from the nodal expression domain (Fig. 3e and [37, 53]). Interestingly, an intermediate scenario is encountered in amphioxus. Here, like in amphibians, a bilateral nodal expression domain was found, but in the anterior part of the archenteron like in sea urchins. The expression on the right side of amphioxus later disappears, possibly due to a leftward cilia-driven flow [3, 26] (Fig. 4b). This reasoning is further supported by the recent description of a dand5 homolog, which was found co-expressed with nodal but down-regulated on the left side, resembling the situation at the vertebrate LRO [28]. Interestingly, a dand5 orthologue was not reported for $P$. lividus, nor was it found in S. purpuratus [53]. Considering just two features, localization of the supposed LRO and presence or absence of dand5, sea urchins, cephalochordates and vertebrates could represent three distinct states, indicative of evolutionary transitions: while sea urchins and amphioxus share an anterior (distal) position of the LRO within the archenteron, amphioxus and vertebrates both possess an orthologue of the Nodal antagonist dand5, but only the vertebrates localize their LRO close to the blastopore. What kind of functional adaptations underlie these variations remains to be investigated.

There are additional LR components worth being considered in the evolutionary context, namely $p k d 2$ and bicc1, both of which were expressed in early sea urchin gastrula mesendodermal tissues during invagination (Fig. 2i-k, Additional file 1: Figure S1 and [41]). bicc1 in mouse and frog functions to polarize LR cilia [40]. In that sense the expression in sea urchins correlated with that in frog and mouse: it is precisely the bicc1 expressing cells 

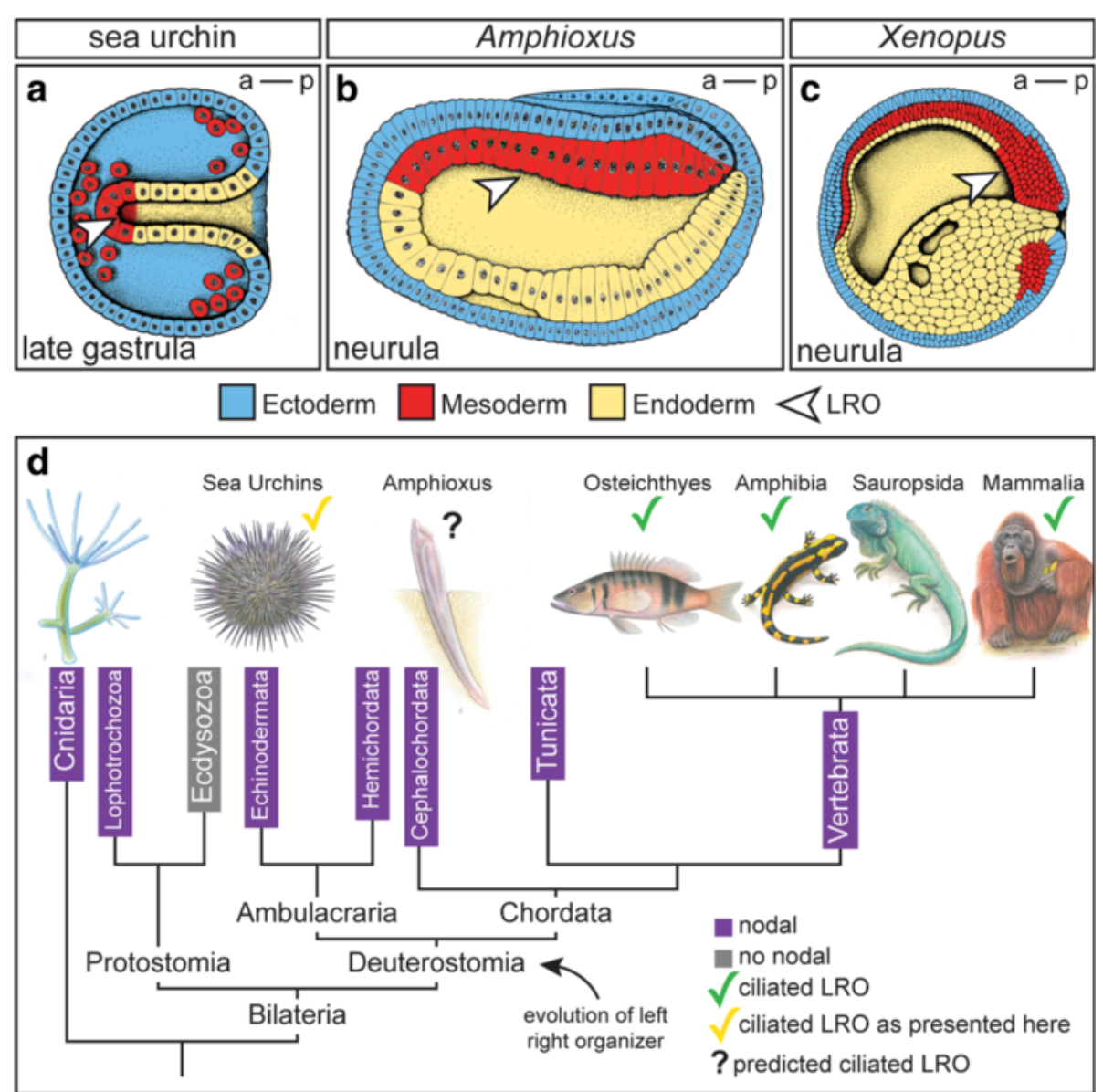

Fig. 4 The ciliated Left-Right Organizer: a synapomorphy of the deuterostomes. a-c Schematic drawings of sea urchin (a), amphioxus (b) and Xenopus (c) gastrula/neurula stage embryos (anterior to the left). In all cases the archenteron harbors cells of mesodermal fate (SM, red), which in frog and sea urchin are ciliated and function as LROs. The position of mesodermal LROs is indicated by an arrowhead. $\mathbf{d}$ nodal is evolutionary conserved among metazoans except for the ecdysozoa. Deuterostomes share a ciliated LRO as a common synapomorphy. Drawings adapted in parts from Blum et al. 2014 [3]

which harbor cilia polarized to the posterior pole in mid gastrula stage embryos in the sea urchin, and which we here report to be motile (cf. Fig. $1 \mathrm{~h}$ and Additional file 1: Figure S1 and Additional file 2: Movie 1). Expression differed in another aspect, however, as bicc1 mRNA was not colocalized with nodal expression at the archenteron tip, different from the vertebrates, where bicc 1 is co-expressed with nodal in the LRO-flanking cells - and might be involved in sensing of flow [40]. It would be interesting to know where bicc1 is expressed in amphioxus, around the blastopore or in the anterior mesoderm.

$p k d 2$ in the sea urchin embryo was expressed in the vegetal mesoderm and endoderm at early to mid-gastrula stages and at the tip of the archenteron. This expression is remarkable, as $p k d 2$ has not been reported to be transcriptionally up-regulated in or at any vertebrate LRO. The encoded protein, the calcium channel Polycystin-2, however, is present in mouse and fish LROs, where it is involved in flow sensing $[19,20,22]$. Functional conservation of symmetry breakage is further supported by the expression of $p k d 2$ in Xenopus gastrula embryos, where it is highly expressed in the mesendodermal ring around the blastopore, reminiscent of the vegetal mesoderm expression in sea urchins (our unpublished observations). Again, expression in amphioxus has not been reported as yet but should be highly informative. Taken together, the presence of cilia and the expression of conserved LR genes strongly argue for a conserved role of motile archenteron cilia in LR symmetry breakage.

The most convincing result, however, is presented by our analysis of cilia function, where deciliation at a time point just prior to asymmetric induction of nodal in the archenteron resulted in altered expression of the Nodal cascade. Molecular asymmetries were lost, with a differential response of nodal and pitx2. While archenteron nodal was mostly absent or below the detection level of the experiment, the ectodermal domain and later on pitx 2 in the coelomic pouches became expanded and bilateral (Fig. 3). These observations cannot be attributed to the absence of archenteron Nodal. When Nodal was 
experimentally manipulated in the archenteron to be absent or expressed in a bilateral manner, the ectodermal domain remained asymmetrical, although in a randomized manner, being activated either on the left or on the right side [33, 37]. The ectoderm is still competent to express nodal on both sides during gastrulation, as shown by Activin treatment of early gastrula embryos [33]. The maintenance of unilateral ectodermal nodal expression might thus be explained by an additional ectodermal cilia function, which was ablated in high salt-treated embryos as well, but we can only speculate on this issue. Our results would be explained if external cilia had a function in restricting Nodal cascade activation unilaterally in the ectoderm, and the archenteron cilia provided a biasing cue, as they do during vertebrate LR axis determination [2].

While this manuscript was under review, two papers were published that dealt with symmetry breaking in sea urchin embryos. In agreement with our conclusions, Takemoto et al. deduced that cilia were required for symmetry breakage in sea urchins. Application of an inhibitor of motile cilia to early blastula stage embryos resulted in a loss of nodal asymmetry, archenteron cilia, however, were not analyzed in this study [54]. Warner et al. (2016) injected 1-cell stage embryos with a kinesin-2 antibody, which removed all cilia from the time point of injection onwards. Nodal was strongly reduced in the four embryos analyzed, while asymmetric SoxE expression in the coelomic pouches was retained in the six embryos included in this one experiment. The authors concluded that cilia were not required for symmetry breakage but rather for hedgehog signaling-mediated nodal maintenance [55]. We strongly disagree with this conclusion for the following reasons: (1) Lepage and colleagues have shown that asymmetric SoxE expression occurs independent of archenteron Nodal; (2) the removal of all cilia from the earliest developmental stages onwards potentially impacts on many more signaling pathways, such as for example hedgehog, as shown by Warner et al (2016) [55], and thus most probably impact on stages before archenteron cilia emerge; (3) attenuated nodal expression by permanent cilia ablation, as described by Warner et al., might be the result of a) loss of archenteron cilia function, which causes bilateral expanded nodal expression as shown in our present work, followed in time by b) loss of hedgehog-mediated nodal maintenance, as described by Warner et al. (2016).

We like to extend our evolutionary considerations to the precursor tissue of the LRO in the sea urchin. Keller and colleagues previously revitalized the concept of the superficial mesoderm (SM) in the chordate lineage. Best characterized in amphibians, the SM of the pre-gastrula embryo gives rise to the archenteron LRO during gastrula/neurula stages to end up in the axial and paraxial mesoderm during later embryogenesis [8]. Besides the birds, which lack an LRO, the SM has been identified in most chordate lineages $[8,11,56]$ (Fig. 4a-d). In the sea urchin mesenchyme blastula, the nonskeletogenic mesoderm, together with the endoderm, locates superficially at the vegetal pole as well, reminiscent of the situation in amphibians [8,57]. Of the known SM marker genes in the frog, foxj1 [58-60], nodal3 [58] and wnt11b [61], only foxj1 has been analyzed in sea urchins (Fig. 2e-g). Its mRNA localization in the early gastrula vegetal cells indeed argues for a conserved blastula stage SM (Fig. 2h). Furthermore, like in vertebrate SM tissues, these cells show accumulation of nuclear $\beta$-catenin at the very time point when the SM is specified [62]. This is of relevance, as foxj1 expression has been shown to be directly induced by canonical Wnt signaling.

\section{Conclusions}

We conclude that the early sea urchin embryo represents an ancestral deuterostome state with a vegetal SM at blastula stages, which transforms into an archenteron LRO, where motile cilia are necessary to break the bilateral symmetry. Cilia-driven symmetry breakage thus should represent a synapomorphy of the deuterostome lineage.

\section{Methods}

\section{Animals and embryo manipulation}

Adult $P$. lividus were collected in Pula/Croatia in early summer during the natural breeding season and reared short-term in the laboratory. Adult S. pallidus were collected at the MSU White Sea Biological Station, Kandalaksha Bay/Russia during the summer. Embryos were obtained by artificial fertilization after injecting adult specimen with $1 \mathrm{ml}$ of 0.5 molar $\mathrm{KCl}$ into the oral field to obtain sperm and oocytes and raised in artificial sea water (ASW) containing antibiotics at $18-20{ }^{\circ} \mathrm{C}$ or $4{ }^{\circ} \mathrm{C}$ for P. lividus and S. pallidus, respectively.

\section{High-salt and Ciliobrevin D treatment}

Early or mid-gastrula stage embryos were transferred to $3 \% \mathrm{ASW}$, which contained high concentrations of $\mathrm{NaCl}$ (1 M) but regular seawater molarities of all other ions, and kept for 60-90 s before washing and transferring to regular ASW. Efficiency of deciliation was controlled in the microscope by absence of embryonic swimming behavior. Subsequently, embryos were incubated until the desired stages for treatment or analysis were reached. For dynein motor inhibition, embryos were transferred to ASW containing $50 \mu \mathrm{M}$ Ciliobrevin D at early gastrula stage and incubated until control untreated specimens reached late gastrula stage. For SB203580 treatment (ENZO Life Sciences), embryos were 
transferred in sterile sea water containing either $20 \mu \mathrm{M}$ or $5 \mu \mathrm{M}$ of SB203580 or DMSO as a control in the same dilution and grown until pluteus stage.

\section{RT-PCR}

Total RNA was isolated from embryos at various stages of development using peqGOLD Trifast (VWR International $\mathrm{GmbH}$, Erlangen/Germany) and cDNAs were prepared using standard protocols. Primers used for PCR amplification of fragments were designed using ESTs:

plbicc1_fwd: 5'gcctgaggtttggttagtgc3', plbicc1_rev: 5' cgatctgtcctgcaatagaaacc 3 ', plfoxj1_fwd: 5' gtcaacacattccaac catctc3', plfoxj1_rev: 5' ${ }^{\prime}$ ctcttctttggcatggtctg3', pldnah9_ fwd: 5' tgccaactttcaatcatatttca3', pldnah9_rev: 5' atgaacactc tacatcagagatc3', plpkd2_fwd: 5' atccctctggagaacgagac3', plpkd2_rev: 5'gcaaacatgacagtgaatcctc3', plnodal_fwd: 5'tt tcttcgctccattcctcc3', plnodal_rev: 5' gaactaagacggctccttcc3'

\section{Cloning of constructs}

Partial coding sequences of plbicc1, plfoxj1, pldnah9, plpkd2 and plnodal were cloned in the pGEM-T Easy vector system and verified by sequencing.

\section{RNA in situ hybridization}

Embryos were fixed in paraformaldehyde (PFA) $4 \%$ PFA for $30 \mathrm{~min}$, stored in $100 \%$ Ethanol at $-20{ }^{\circ} \mathrm{C}$ and processed following standard protocols. Digoxigenin-labeled RNA (Roche) probes were prepared with SP6 or T7 RNA polymerase on linearized pGEM-T Easy templates (Promega). In situ hybridization was modified from [63]. Initial rehydration steps were performed with $1 \%$ BSA in PBS to avoid agglutination of embryos. All steps were performed either in glass wells or custom made plastic tubes with meshwork.

\section{Immunofluorescence}

Embryos were fixed in $4 \%$ PFA in ASW for $30 \mathrm{~min}$, washed with PBS, permeabilized with PBS/0.1 \% Triton X-100 (Sigma), blocked with CAS-Block ${ }^{\mathrm{Tm}}$ Histochemical Reagent (Life technologies) and incubated with primary antibodies overnight at $4{ }^{\circ} \mathrm{C}$. Secondary antibodies were incubated for $2 \mathrm{~h}$ at room temperature. Primary antibodies used in this study were: anti-acetylated $\alpha$-tubulin (Sigma, T6793); anti- $\alpha$-tubulin (Sigma, T6199). Reagents used for visualization of nuclei included DAPI, Hoechst and propidium iodide. To visualize cell membranes, Phalloidin-Tetramethylrhodamin B isothiocyanate (Sigma, P1951) or Alexa 488 Phalloidin (Life technologies, A12379) were used.

\section{Scanning electron microscopy}

Wild-type or HS-treated embryos were fixed in $4 \%$ PFA containing $0.2 \%$ Glutaraldehyde in PBS. Preparation of embryos for SEM followed standard protocols. Prior to sputter-coating with gold, some embryos were manually broken using a pipette tip, in order to visualize the archenteron surface.

\section{High-speed videography of cilia motility in gastrula stage embryos}

To observe ciliary motility directly, gastrula stage embryos were either transferred into a solution containing 0.5$1.0 \%$ methylcellulose (Sigma) in ASW in order to slow down beating of the cilia in the forming archenteron cavity (for early to mid-gastrula stages), or positioned within a nitex screen (SEFAR, Germany) to focus on the central archenteron cavity (mid-late gastrula stages). Imaging was performed using a Hamamatsu ORCA-Flash 4.0 Digital CMOS camera mounted on a Zeiss Imager.M1 microscope equipped with a Plan-Apochromat $100 x / 1.4$ oil objective. Acquisition of frames was performed using the Zeiss ZEN software. Fiji [64] was used for temporal difference imaging, i.e. for visualization of movements against a 'static' background. Each frame of a time-series was subtracted from its consecutive frame $\left(t_{n}-t_{n+1}\right)$, resulting in different pixel grey values with black indicating no change in between two frames.

\section{Photo-documentation and picture analysis}

IF pictures were taken on a Zeiss Observer. Z1/LSM 700 equipped with a $63 \mathrm{x}$ objective (C-Apochromat $63 \mathrm{x} / 1.2 \mathrm{~W}$ Corr). Photographs of embryos after in situ hybridization were taken on an Axioskop 2 mot plus (Zeiss, Germany) and processed in Adobe Photoshop. Figures were assembled using Adobe Illustrator.

\section{Additional files}

Additional file 1: Figure S1. mRNA expression of biccl (a-d) and overall normal development of Paracentrotus lividus embryos upon high salt induced deciliation (e-j) or upon early MAPK/p38 inhibition (k-n). (PDF 877 kb)

Additional file 2: Movie 1. Archenteron cilia are motile. Real-time movie of early to mid gastrula stage embryo in lateral-vegetal view reveals fast movement of polarized cilia on laterally invaginating cells at the posterior aspect of the archenteron (cf. Fig. 1h). Bright-field movie (a) and high-magnification (white box) of the inner archenteron wall (a'). (b) Temporal difference imaging rendered version of the same movie, which highlights pixel grey value differences between two consecutive frames. Movie was acquired at 55fps (cf. methods for details). (AVI $8410 \mathrm{~kb}$ )

Additional file 3: Movie 2. Monocilia inside the archenteron of gastrula stage embryos rotate Real-time movie of mid to late gastrula stage embryo in vegetal view, focusing on the central archenteron region, reveals fast rotating monocilia inside the archenteron (cf. Fig. 1g). (a) bright-field movie. (b) Temporal difference imaging rendered version of the same movie, which highlights pixel grey value differences between two consecutive frames. (a', b') High-magnification area as outlined in (a, b). Movie was acquired at $35 \mathrm{fps}$. Please note the rotating cilia on the inner epithelium of this embryo. (AVI $7326 \mathrm{~kb}$ )

Acknowledgements

We thank Cathrin Hagenlocher for SEM assistance and Nikoloz Tsikolia for advice. Work in the Blum lab was supported by DFG grants BL285/9-2 and BL285/10-1. M. Tisler was funded by the Federal Ministry of Education and 
Research (01PL11003), Humboldt reloaded at the University of Hohenheim, Germany. S. Kremnyov was funded by a RFBR fund, grant 14-04-32085 mol_a.

\section{Authors' contributions}

PV, MB, TT, MT and SK designed experiments. MT, FW, SM, SK, TT and PV performed experiments. PV, MB, AS, TT and SK interpreted the results. PV and $\mathrm{MB}$ wrote the manuscript. All authors reviewed the manuscript. All authors read and approved the final manuscript

\section{Competing interests}

The authors declare that they have no competing interests.

\section{Ethics approval and consent to participate}

Not applicable.

\section{Author details}

'University of Hohenheim, Institute of Zoology, 70593 Stuttgart, Germany. ${ }^{2}$ Department of Embryology, Lomonosov Moscow State University, Moscow, Russia. ${ }^{3}$ Present Address: Centre for Organismal Studies, Im Neuenheimer Feld 230, Heidelberg University, 69120 Heidelberg, Germany.

Received: 3 February 2016 Accepted: 29 July 2016

Published online: 23 August 2016

\section{References}

1. Dunn CW, Giribet G, Edgecombe GD. Animal Phylogeny and Its Evolutionary Implications. Annu Rev Ecol Syst. 2014;45:371-95.

2. Yoshiba S, Hamada H. Roles of cilia, fluid flow, and $\mathrm{Ca} 2+$ signaling in breaking of left-right symmetry. Trends Genet. 2014;30:10-7.

3. Blum M, Feistel K, Thumberger T, Schweickert A. The evolution and conservation of left-right patterning mechanisms. Development. 2014; 141:1603-13.

4. Hamada $\mathrm{H}$. Breakthroughs and future challenges in left-right patterning. Development Growth Diff. 2008:50:571-8.

5. Hirokawa N, Tanaka Y, Okada Y. Cilia, KIF3 molecular motor and nodal flow. Curr Opin Cell Biol. 2012:24:31-9.

6. Blum $\mathrm{M}$, et al. Ciliation and gene expression distinguish between node and posterior notochord in the mammalian embryo. Differentiation. 2007;75:133-46

7. Sulik K, et al. Morphogenesis of the murine node and notochordal plate. Dev Dyn. 1994;201:260-78.

8. Shook DR, Majer C, Keller R. Pattern and morphogenesis of presumptive superficial mesoderm in two closely related species, Xenopus laevis and Xenopus tropicalis. Dev Biol. 2004;270:163-85.

9. Nonaka S, et al. De novo formation of left-right asymmetry by posterior tilt of nodal cilia. PLoS Biol. 2005;3:e268.

10. Schweickert $A$, et al. Cilia-Driven Leftward Flow Determines Laterality in Xenopus. Curr Biol. 2007;17:60-6.

11. Blum M, Weber T, Beyer T, Vick P. Evolution of leftward flow. Semin Cell Dev Biol. 2009:20:464-71.

12. Kramer-Zucker AG. Cilia-driven fluid flow in the zebrafish pronephros, brain and Kupffer's vesicle is required for normal organogenesis. Development. 2005;132:1907-21

13. Okada Y, Takeda S, Tanaka Y, Izpisúa-Belmonte JC, Hirokawa N. Mechanism of nodal flow: a conserved symmetry breaking event in left-right axis determination. Cell. 2005:121:633-44.

14. Nonaka S, et al. Randomization of left-right asymmetry due to loss of nodal cilia generating leftward flow of extraembryonic fluid in mice lacking KIF3B motor protein. Cell. 1998;95:829-37.

15. Essner JJ. Kupffer's vesicle is a ciliated organ of asymmetry in the zebrafish embryo that initiates left-right development of the brain, heart and gut. Development. 2005;132:1247-60.

16. Schweickert, A. et al. The Nodal Inhibitor Coco Is a Critical Target of Leftward Flow in Xenopus. Curr Biol. 2010;20:738-43.

17. Hojo M, et al. Right-elevated expression of charon is regulated by fluid flow in medaka Kupffer's vesicle. Development Growth Diff. 2007:49:395-405.

18. Marques $\mathrm{S}$, et al. The activity of the Nodal antagonist Cerl-2 in the mouse node is required for correct L/R body axis. Genes Dev. 2004;18:2342-7.

19. Yuan S, Zhao L, Brueckner M, Sun Z. Intraciliary calcium oscillations initiate vertebrate left-right asymmetry. Curr Biol. 2015;25:556-67.
20. Pennekamp $P$, et al. The ion channel polycystin-2 is required for left-right axis determination in mice. Curr Biol. 2002;12:938-43.

21. Schottenfeld J, Sullivan-Brown J, Burdine RD. Zebrafish curly up encodes a $\mathrm{Pkd} 2$ ortholog that restricts left-side-specific expression of southpaw. Development. 2007;134:1605-15

22. Yoshiba $\mathrm{S}$, et al. Cilia at the node of mouse embryos sense fluid flow for left-right determination via Pkd2. Science. 2012;338:226-31.

23. Namigai EKO, Kenny NJ, Shimeld SM. Right across the tree of life: the evolution of left-right asymmetry in the Bilateria. Genesis. 2014;52:458-70.

24. Chea HK, Wright CV, Swalla BJ. Nodal signaling and the evolution of deuterostome gastrulation. Dev Dyn. 2005;234:269-78.

25. Duboc V, Lepage T. A conserved role for the nodal signaling pathway in the establishment of dorso-ventral and left-right axes in deuterostomes. J Exp Zool. 2007;310B:41-53.

26. Yu J-K, Holland LZ, Holland ND. An amphioxus nodal gene (AmphiNodal) with early symmetrical expression in the organizer and mesoderm and later asymmetrical expression associated with left-right axis formation. Evol Dev. 2002;4:418-25.

27. Morokuma J, Ueno M, Kawanishi H, Saiga H, Nishida H. HrNodal, the ascidian nodal-related gene, is expressed in the left side of the epidermis, and lies upstream of HrPitx. Dev Genes Evol. 2002;212:439-46.

28. Le Petillon Y, Oulion S, Escande M-L, Escriva H, Bertrand S. Identification and expression analysis of BMP signaling inhibitors genes of the DAN family in amphioxus. Gene Expr Patterns. 2013;13:377-83.

29. Boorman CJ, Shimeld SM. Pitx homeobox genes in Ciona and amphioxus show left-right asymmetry is a conserved chordate character and define the ascidian adenohypophysis. Evol Dev. 2002;4:354-65.

30. Onai T, YU J-K, Blitz IL, Cho KWY, Holland LZ. Opposing Nodal/Vg1 and BMP signals mediate axial patterning in embryos of the basal chordate amphioxus. Dev Biol. 2010;344:377-89.

31. Yasui $K$, Zhang $S$, Uemura M, Saiga $H$. Left-right asymmetric expression of BbPtx, a Ptx-related gene, in a lancelet species and the developmental leftsidedness in deuterostomes. Development. 2000;127:187-95.

32. Soukup $V$, et al. The Nodal signaling pathway controls left-right asymmetric development in amphioxus. EvoDevo. 2015;6:1-22.

33. Duboc V, Röttinger E, Lapraz F, Besnardeau L, Lepage T. Left-right asymmetry in the sea urchin embryo is regulated by nodal signaling on the right side. Dev Cell. 2005:9:147-58.

34. McClay DR. Evolutionary crossroads in Dev. Biol.: sea urchins. Development. 2011:138:2639-48.

35. Molina MD, de Crozé N, Haillot E, Lepage T. Nodal: master and commander of the dorsal-ventral and left-right axes in the sea urchin embryo. Curr Opin Genet Dev. 2013:23:445-53.

36. Su Y-H. Telling left from right: Left-right asymmetric controls in sea urchins, Genesis. 2014:52:269-78.

37. Bessodes $\mathrm{N}$, et al. Reciprocal signaling between the ectoderm and a mesendodermal left-right organizer directs left-right determination in the sea urchin embryo. PLoS Genet. 2012;8:e1003121.

38. Christiaen $\mathrm{L}$, et al. Evolutionary modification of mouth position in deuterostomes. Sem Cell Dev Biol. 2007;18:502-11.

39. Lapraz F, Haillot E, Lepage T. A deuterostome origin of the Spemann organiser suggested by Nodal and ADMPs functions in Echinoderms. Nat Commun. 2015:6:8434.

40. Maisonneuve $\mathrm{C}$, et al. Bicaudal C, a novel regulator of Dvl signaling abutting RNA-processing bodies, controls cilia orientation and leftward flow. Development. 2009;136:3019-30.

41. Yaguchi S, Yaguchi J, Inaba K. Bicaudal-C is required for the formation of anterior neurogenic ectoderm in the sea urchin embryo. Sci Rep. 2014;4:6852-7.

42. Firestone AJ, et al. Small-molecule inhibitors of the AAA+ ATPase motor cytoplasmic dynein. Nature. 2012;484:125-9.

43. Auclair W, Siegel BW. Cilia regeneration in the sea urchin embryo: evidence for a pool of ciliary proteins. Science. 1966:154:913-5.

44. Lancaster MA, Gleeson JG. The primary cilium as a cellular signaling center: lessons from disease. Curr Opin Genet Dev. 2009:19:220-9.

45. Warner JF, McCarthy AM, Morris RL. Hedgehog signaling requires motile cilia in the sea urchin. Mol Biol Evol. 2013;31:18-22.

46. Eldon ED, Angerer LM, Angerer RC, Klein WH. Spec3: embryonic expression of a sea urchin gene whose product is involved in ectodermal ciliogenesis. Genes Dev. 1987;1:1280-92.

47. Kinukawa M, Vacquier VD. Adenylate kinase in sea urchin embryonic cilia. Cell Motil Cytoskeleton. 2007;64:310-9. 
48. Prulière G, Cosson J, Chevalier S, Sardet C, Chenevert J. Atypical protein kinase C controls sea urchin ciliogenesis. Mol Biol Cell. 2011;22:2042-53.

49. Stephens RE. Tubulin and tektin in sea urchin embryonic cilia: pathways of protein incorporation during turnover and regeneration. J Cell Sci. 1994; 107(Pt 2):683-92.

50. Stephens RE. Differential protein synthesis and utilization during cilia formation in sea urchin embryos. Dev Biol. 1977;61:311-29.

51. Shen MM. Nodal signaling: developmental roles and regulation. Development. 2007;134:1023-34

52. Bradham CA, McClay DR. p38 MAPK is essential for secondary axis specification and patterning in sea urchin embryos. Development. 2006;133:22-32.

53. Luo Y-J, Su Y-H. Opposing nodal and BMP signals regulate left-right asymmetry in the sea urchin larva. PLoS Biol. 2012;10:e1001402.

54. Takemoto A, Miyamoto T, Simoni F, Kurogi N, Shirae-Kurabayashi M, Awazu A, Suzuki KT, Yamamoto T, Sakamoto N. Cilia play a role in breaking leftright symmetry of the sea urchin embryo. Genes Cells. 2016;21(6):568-78.

55. Warner JF, Miranda EL, McClay DR. Contribution of hedgehog signaling to the establishment of left-right asymmetry in the sea urchin. Dev Biol. 2016; 411(2):314-24

56. Männer J. Does an equivalent of the 'ventral node' exist in chick embryos? A scanning electron microscopic study. Anat Embryol. 2001;203:481-90.

57. Schweickert A, Walentek P, Thumberger T, Danilchik M. Linking early determinants and cilia-driven leftward flow in left-right axis specification of Xenopus laevis A theoretical approach. Differentiation. 2011;83(S77-87):1-11.

58. Beyer T, et al. Serotonin Signaling Is Required for Wnt-Dependent GRP Specification and Leftward Flow in Xenopus. Curr Biol. 2012;22:33-9.

59. Stubbs JL, Oishi I, Izpisúa-Belmonte JC, Kintner C. The forkhead protein Foxj1 specifies node-like cilia in Xenopus and zebrafish embryos. Nat Genet. 2008;40:1454-60

60. Blum M, Schweickert A, Vick P, Wright CVE, Danilchik MV. Symmetry breakage in the vertebrate embryo: When does it happen and how does it work? Dev Biol. 2014;393:109-23.

61. Walentek P, Schneider I, Schweickert A, Blum M. Wnt1 1b Is Involved in CiliaMediated Symmetry Breakage during Xenopus Left-Right Development. PLOS ONE. 2013;8:e73646-9.

62. Logan CY, Miller JR, Ferkowicz MJ, McClay DR. Nuclear beta-catenin is required to specify vegetal cell fates in the sea urchin embryo. Development. 1999;126:345-57.

63. Belo JA, et al. Cerberus-like is a secreted factor with neutralizing activity expressed in the anterior primitive endoderm of the mouse gastrula. Mech Dev. 1997;68:45-57.

64. Schindelin J, Arganda-Carreras I, Frise E, et al. Fiji: an open-source platform for biological-image analysis. Nat Methods. 2012;9:676-82.

\section{Submit your next manuscript to BioMed Central and we will help you at every step:}

- We accept pre-submission inquiries

- Our selector tool helps you to find the most relevant journal

- We provide round the clock customer support

- Convenient online submission

- Thorough peer review

- Inclusion in PubMed and all major indexing services

- Maximum visibility for your research

Submit your manuscript at www.biomedcentral.com/submit

C Biomed Central 